\title{
Perus Bevölkerung: Das historische Gewordensein von Diskriminierungen und Privilegierungen ${ }^{1}$
}

\author{
MARTINA NEUBURGER \\ Universität Hamburg Institut für Geographie \\ KATRIN SINGER \\ Universität Hamburg Institut für Geographie
}

\begin{abstract}
Kurzfassung
Die Bevölkerungs- und Sozialstruktur Perus zeigt eine große Vielfalt, die sich erst aus einer Darstellung des Gewordenseins gesellschaftlicher Strukturen und den Verwobenheiten von soziopolitischen Prozessen in unterschiedlichen räumlichen und zeitlichen Dimensionen erschließt. Die bereits in der inkaischen Gesellschaft angelegten Hierarchien wurden in der Kolonialzeit nicht gebrochen, sondern von der spanischen Krone reproduziert und mit eigenen Machtstrukturen überlagert. Die Systeme der Feudalgesellschaft mit Leibeigenschaft und Sklaverei wirken bis heute nach. Die hierarchischen Beziehungen zwischen den unterschiedlichen ethno-sozialen Gruppen sind bis heute durch die verschiedensten Formen von Diskriminierung und Rassismen wirkmächtig, wenn es um den Zugang zu Land und Einkommen, Bildung und gesellschaftliche Anerkennung geht. Anhand zweier Fallstudien arbeitet der Beitrag gesellschaftliche Strukturierungsdynamiken als Ergebnis ungleicher Machtstrukturen und postkolonialer Verhältnisse exemplarisch heraus. Das erste Beispiel fokussiert auf den Callejón de Huaylas im Department Ancash und setzt sich mit denjenigen Diskriminierungsmechanismen auseinander, unter denen insbesondere die ländlich-andine Bevölkerung leidet. Das zweite Beispiel konzentriert sich auf die ehemalige deutsch-tirolische Siedlung Pozuzo im Department Pasco, in dem die europä-ischstämmige Bevölkerung als privilegierte Bevölkerungsgruppe ihre migrantische Identität machtvoll rekonstruiert und damit andere Zuschreibungen oder Hybriditäten negiert. In beiden Fallstudien spielen ethnische Zuschreibungen als Differenzkategorie und Legitimierungsstrategie eine wichtige Rolle für die Herausbildung der aufgezeigten Ungleichheiten.
\end{abstract}

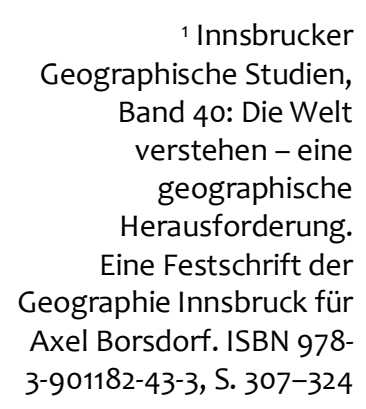




\title{
Peru's Population: The historical evolution of discriminations and privileges
}

\begin{abstract}
The social and population structure of Peru is highly diverse and can only be understood via the emergence of social structures and the interdependence of socio-political processes in various spatial and temporal dimensions. The hierarchies already established within the Inca society were not broken during colonial times, but reproduced by the Spanish crown and superimposed with their own power structures. The systems of the feudal society, with serfdom and slavery, continue to have an effect today. The hierarchical relations between the different ethnosocial groups retain their potency via various forms of discrimination and racism in terms of access to land and income, education and social acceptance. By looking at two case studies, this essay identifies typical social structuring dynamics as the result of unequal power structures and postcolonial circumstances. The first example focuses on the Callejón de Huaylas in Ancash Department and the mechanisms of discrimination suffered by the rural Andean population in particular. The second example concentrates on the formerly German-Tyrolean settlement of Pozuzo in Pasco Department, where the European-origin population, as a privileged group, reconstructs its migrant identity as powerful, thus denying other attributions or hybridities. In both case studies ethnic attributions play an important role as differentiation category and legitimation strategy for the emergence of the presented inequalities.
\end{abstract}

\section{O povo peruano: a evolução histórica de discriminações e privilégios}

\section{Resumo}

A estrutura popular e social do Peru indica uma grande diversidade, que se mostra primeiramente na evolução das estruturas sociais e do entrelaçamento de processos sociopolíticos em diferentes dimensões espaciais e temporais. As hierarquias já estabelecidas na sociedade inca não foram quebradas durante o período colonial, mas sim, reproduzidas pela coroa espanhola e sobreposicionadas com as próprias estruturas de poder. O sistema da sociedade feudal com servidão e escravidão ainda surte efeito até os dias de hoje. As relações hierárquicas entre os diferentes grupos étno-sociais ainda são poderosos através de várias formas de discriminação e racismo, quando se trata de acesso à terra, à renda, à educação e reconhecimento social. Através de dois estudos de caso, são relatadas exemplarmente as estruturas sócio-dinâmicas de resultados, as estruturas desiguais e relações pós-coloniais. O primeiro exemplo foca o Callejón de Huaylas no departamento Ancach e analisa os mecanismos de discriminação sobre quais especialmente a população colonial dos andes sofre. $O$ segundo exemplo se concentra no antigo assentamento germano-tiroles Popuzo, no departamento de Pasco, no qual a população de origem europeia, como a classe mais privilegiada, reconstrói poderosamente sua identidade imigrante, e assim, nega outras atribuições ou hibridades. Em ambos estudos, as atribuições étnicas desempenham um papel importante para a formação das desigualdades indicadas.

\section{Einführung}

Peru gilt unter den südamerikanischen Ländern gemeinhin als „typisches“ Andenland mit einem hohen Anteil an indigener Bevölkerung im Altiplano und einer gleichzeitigen politischen und wirtschaftlichen Dominanz einer mestizischen Minderheit. Bei genauerem Hinsehen lässt sich diese einfache Dichotomie jedoch nicht aufrecht erhalten, denn die Bevölkerungs- und Sozialstruktur Perus zeigt eine große Vielfalt, die sich erst aus einer Darstellung des Gewordenseins 
gesellschaftlicher Strukturen und den Verwobenheiten von soziopolitischen Prozessen in unterschiedlichen räumlichen und zeitlichen Dimensionen erschließt. Vorkolumbische Rahmenbedingungen, Umwälzungen im Zuge der Eroberung durch die europäischen Kolonialmächte, Unabhängigkeit, Militärdiktaturen, Terrorismus und Demokratisierungen sowie aktuelle Globalisierungstendenzen bilden in der peruanischen Geschichte wichtige Elemente, die sowohl Brüche, aber auch Kontinuitäten in der gesellschaftlichen Entwicklung hervorgebracht haben und die sich noch heute in der Bevölkerungs- und Sozialstruktur Perus widerspiegeln. Insbesondere sozioökonomische Ungleichheiten und Hierarchien sind bis heute eng geknüpft an ethnische Diff erenzen, die gesellschaftlich und politisch immer wieder reproduziert werden und zu vielfältigen und intersektionalen Diskriminierungsformen gegenüber jenen Teilen der Bevölkerung führt, die nicht der mestizischen Norm entsprechen. Persistente Privilegierungsmechanismen für Mestiz*innen und sich als weiß bezeichnende Gruppen ist eine daraus resultierende Entwicklung.

Der folgende Beitrag geht zunächst auf zentrale Dimensionen sozialer Ungleichheiten der Bevölkerung Perus ein, um darauf aufbauend gesellschaftliche Strukturierungsdynamiken als Ergebnis ungleicher Machtstrukturen und postkolonialer Verhältnisse exemplarisch anhand zweier Fallstudien herauszuarbeiten. Das erste Beispiel fokussiert auf den Callejón de Huaylas im Department Ancash und setzt sich mit denjenigen Diskriminierungsmechanismen auseinander, unter denen insbesondere die ländlich-andine Bevölkerung leidet. Das zweite Beispiel konzentriert sich auf die ehemalige deutsch-tirolische Siedlung Pozuzo im Department Pasco, in dem die europäisch-stämmige Bevölkerung als privilegierte Bevölkerungsgruppe ihre migrantische Identität machtvoll rekonstruiert und damit andere Zuschreibungen oder Hybriditäten negiert. In beiden Fallstudien spielen ethnische Zuschreibungen als Diff erenzkategorie und Legitimierungsstrategie eine wichtige Rolle für die Herausbildung der aufgezeigten Ungleichheiten. Damit knüpft der Beitrag an Arbeiten von Axel Borsdorf an, der sich in einigen seiner Studien mit der Rolle europäisch-stämmiger Bevölkerung in unterschiedlichen südamerikanischen Ländern beschäftigt hat (Borsdorf 1986, 1995, 1997, 2007).

\section{Ungleichheiten in der peruanischen Bevölkerung}

Die Bevölkerung von Peru ist nicht nur in wirtschaftlicher und sozialer Hinsicht sehr vielfältig, sondern zeichnet sich auch durch eine ethnisch-kulturelle und politische Heterogenität aus. Diese Vielfalt kann verstanden werden als ein Ergebnis zeitlich und räumlich diff erenzierter Dynamiken von natürlicher Bevölkerungsentwicklung, von Zu- und Ab- bzw. Auswanderung, von Vermischung (mestizaje) und Assimilation, aber auch von Genozid, Vertreibung und Verdrängung. Die Vielschichtigkeit der damit verbundenen Prozesse darzustellen, ist an dieser Stelle nicht möglich (GolashBoza 2011, Henk 2010). Es soll dennoch versucht werden, die wichtigsten Dimensionen gesellschaftlicher Hierarchisierungen in ihrem historischen Kontext schlaglichtartig zu beleuchten.

Hinsichtlich des historischen Gewordenseins der peruanischen Bevölkerung deuten bereits archäologische Funde auf eine Vielzahl von Kulturen und Ethnien 
sowohl in vorinkaischer als auch in inkaischer Zeit hin (Herrera 2007, Golte 2005, zahlreiche Beiträge in Boletín de Arqueología PUCP 2002, 2003, 2004). Dabei stand die Bevölkerung der andinen Regionen in regem Austausch mit den amazonischen Gruppen. Mit Beginn der Kolonialzeit kamen sowohl europäische Gruppen durch die Kolonisator*innen selbst, als auch afrikanische Bevölkerung als Sklav*innen hinzu (Golash-Boza 2011). Durch die Kolonialisierung und systematische Ermordung indigener Bevölkerung einerseits sowie durch die weitere Zuwanderung aus Europa und Asien und das natürliche Bevölkerungswachstum verbunden mit interkulturellen Partnerschaften andererseits verschob sich die ethnische Struktur, so dass die Konquistador*innen im Jahr 1792 die damals als ,indigen“ bezeichnete Bevölkerung nur noch auf 56 \% der Gesamtbevölkerung schätzten (Quiroz Chueca 2007, Hensel \& Potthast 2013: 266). Dabei ist allerdings unklar, ob die amazonischen Bevölkerungsgruppen in diese Schätzung mit eingingen.

Sowohl die spanische, als auch die mestizische Bevölkerung wuchs in den Jahrhunderten der Kolonialzeit langsam, aber stetig an, während die indigene Bevölkerung des Landes aufgrund von Verfolgung, Arbeitsbelastung, eingeschleppten Krankheiten etc. zunehmend zu einer ethnischen Minderheit im eigenen Land wurde (Newson 2006, Brea 2003). Die katholische Kirche trug mit der Missionierung aller Gruppen zu einer gewissen kulturellen Homogenisierung bei, auch wenn sich dadurch ein Synkretismus entwickelte, der noch heute in Riten und Zeremonien verschiedener comunidades zu fi nden ist. In den letzten Jahren verlangen die stark expandierenden evangelikalen Kirchen eine bedingungslose Zuwendung zu christlichen Glaubensformen, die oft jegliche Form des Synkretismus ablehnen (Rösing 2001, Spedding 2008, Griffi ths 1996).

Nach der Unabhängigkeit von der spanischen Krone im Jahr 1824 und der Abschaffung der Sklaverei im Jahr 1854 warb der junge Nationalstaat billige Arbeitskräfte aus Asien - vor allem aus China und Japan - für die landwirtschaftlichen Kerngebiete der Anden an und lockte junge Familien aus den damaligen Armutsgebieten Mitteleuropas mit dem Angebot von Landeigentum in die dünn besiedelten Gebiete Amazoniens (siehe Borsdorf \& Stadel 2013, Neuburger \& Steinicke 2013). Diese neuen Einwanderungswellen des 19. und 20. Jahrhunderts führten dazu, dass verschiedene historische und sozio-politische Kontexte gleichsam nach Peru importiert wurden und damit zum heutigen Bild der Bevölkerung Perus beitrugen. Die unterschiedlichen Gruppen an Zuwander*innen kamen nicht nur aus unterschiedlichen naturräumlichen Kontexten, sondern brachten auch verschiedene soziale Herkünfte, ökonomische Ressourcen und politische Überzeugungen mit und trafen in Peru wiederum auf sehr vielfältige Strukturen und Prozesse, in die sie sich einfügen mussten. Während die europäischen Siedler*innen von Anfang an ein gesellschaftlich hohes Ansehen aufgrund ihrer Herkunft und Hautfarbe genossen, galten die asiatischen Einwander*innen als sozial marginalisierte Gruppe, die extreme rassistische Diskriminierung erdulden musste (Onken 2013, Hensel 2013).

Die internationale Immigration hat sich nach der ersten Hälfte des 20. Jahrhunderts ins Gegenteil verkehrt. Seit den 1960er Jahren wächst der Strom der peruanischen Auswander*innen stetig an. Neben den USA stellen die wohlhabenderen Nachbarstaaten in Lateinamerika die Hauptmigrationsziele dar. Dort fi nden Peruaner*innen vor allem als Saisonarbeiter*innen in den 
Bewässerungsoasen Chiles und Argentiniens oder als billige Arbeitskräfte in der Textilindustrie des brasilianischen Südostens äußerst prekäre Anstellungen, die teilweise sklavenähnliche Bedingungen annehmen (Rodríguez Vignoli 2004, Silva 2003). Nicht selten bauen Peruaner*innen jedoch im Laufe der Jahre eigene Kleinunternehmen im Ausland auf und halten auch über viele Jahrzehnte hinweg die Kontakte zu ihren Familien aufrecht. Durch diese sehr aktiven, grenzüberschreitenden Netzwerke bleiben die Auswander*innen auch weiterhin ökonomisch - über Rimessen - und sozial - durch die Vermittlung von Kontakten für weitere Auswanderungswillige und über die neuen sozialen Medien - in der Herkunftsregion präsent. Gerade der Zugang zu Rimessen, der meist nur für einige wenige Familien in ländlichen Gemeinden Perus besteht, führt mitunter zu wachsenden Ungleichheiten und sozialen Spannungen in einzelnen comunidades, wenn nicht tradierte Umverteilungsregeln dies verhindern (Acosta et al. 2006, 2008).

Die Verteilung der Bevölkerung auf das peruanische Staatsgebiet weist sehr große räumliche Disparitäten auf, die sich in jüngerer Zeit zum Teil verschieben. Während sich noch in der Kolonialzeit und in den ersten Jahrzehnten der Unabhängigkeit das größte Bevölkerungskontingent im fruchtbaren, rohstoff - und wasserreichen andinen Hochland befand, verschob sich der Bevölkerungsschwerpunkt im Laufe des 20. Jahrhunderts in die Wüstenzonen der Küstenregion. Mit einem Bevölkerungsanteil von rund 13\% im Jahr 2007 waren und sind die amazonischen Departments bis heute die mit Abstand am wenigsten dicht besiedelten Gebiete, obwohl sie einen Anteil von $60 \%$ am gesamten Staatsgebiet Perus ausmachen (INEI 2007). Diese Diskrepanz wird von den verschiedensten Akteuren immer wieder als Legitimation herangezogen, um einerseits natürliche Ressourcen - mineralische Rohstoff e, Erdöl, Wasser, Holz etc. - für die Wirtschaften der dichter besiedelten Regionen abzuziehen oder andererseits um Bevölkerung aus den Andenregionen in Amazonien anzusiedeln. Beides führt regelmä- ßig zu teils gewaltvollen Konfl ikten zwischen den verschiedenen beteiligten Akteuren (siehe zum Beispiel Stetson 2012, Finer et al. 2008, Perz et al. 2005).

Die Verschiebung des regionalen Schwerpunktes der Bevölkerung hat nicht zuletzt mit den Verstädterungsprozessen der letzten Jahrzehnte zu tun. Bis in die 1960er Jahre lag der Schwerpunkt der peruanischen Bevölkerung eindeutig im ländlichen Raum, auch wenn die Stadt-Land-Migration schon in den $1940 e r$ Jahren begonnen hatte, begann mit dem Aufkommen der Guerillabewegung Sendero Luminoso in ländlichen Räumen Ende der 1960er Jahre eine Landfl ucht in die städtischen Zentren Perus, allen voran nach Lima (Huber \& Steinhauf 1997). Die extreme Konzentration von Bevölkerung, Wirtschaftskraft und politischer Macht auf Lima führt dazu, dass gesellschaftliche Normen, politische Programme und wirtschaftliche Entwicklungsziele von den ökonomischen und politischen Eliten im Kontext der Hauptstadt entworfen werden, so dass Lima in gewisser Weise als Maß aller Dinge gilt und andere, besonders ländliche Entwicklungswege oder Lebensentwürfe tendenziell marginalisiert werden (Klarén 2004, Fuenzalida 2009, Onken 2013). Durch Nachahmungsversuche städtischer Lebensformen lässt sich in den wenigsten Kleinstädten zwar eine urbane Verdichtung beobachten. Gleichwohl setzen sich auch in ländlichen Siedlungen - völlig unabhängig von ihrer 
Bevölkerungszahl - urbane Lebensstile und moderne Wirtschaftsformen der mestizischen Norm durch, da sie durch ihre diskursive Dominanz in der peruanischen Gesellschaft sowie durch moderne Kommunikationsmedien auch periphere Regionen erreicht.Insgesamt haben die nationalen und internationalen Wanderungsbewegungen dazu beigetragen, dass einzelne Regionen Perus durch spezifi sche ethnosoziale Strukturen gekennzeichnet sind. Die Küstenregion mit der Metropole Lima bildet das Gebiet mit der höchsten Verstädterungsrate. Der Anteil der weißen und mestizischen Bevölkerung ist höher als in anderen Regionen, denn diese bilden weitgehend die nationalen Eliten in Industrie und Politik, die in den großen Städten an der Küste konzentriert sind. Die Altiplano-Region weist eine hohe Bevölkerungsdichte auf, allerdings mit stärker ländlich-landwirtschaftlicher Prägung. Hier ist der Anteil Quechua- und Aymara-sprechender Bevölkerung relativ hoch, während hacendadxs, caudillxs und Bergbauunternehmer*innen die Eliten der Region bilden. Schließlich repräsentieren die amazonischen Departments die am dünnsten besiedelten Gebiete, in denen die indigenen Gruppen des Tiefl andes einen relativ hohen Anteil aufweisen. Im Zuge der staatlich vorangetriebenen Agrarkolonisation der 1960er und 1970er Jahre wurden zahlreiche campesinxs aus der Andenregion in amazonischen Departments angesiedelt, so dass sich eine ethnische Hierarchie herausgebildet hat, in der die campesinxs die gesellschaftlich höher positionierte Gruppe gegenüber den amazonisch-indigenen Gruppen darstellen.

Die skizzierten Hierarchien in der peruanischen Gesellschaft lassen sich in zahlreichen Bereichen und Kontexten wiederfi nden. Im Folgenden sollen die Diskriminierungsund Privilegisierungsmechanismen an den beiden Beispielen des andinen Callejón de Huaylas und des amazonischen Pozuzo näher erläutert werden.

\section{Diskriminierungsformen im Callejón de Huaylas}

Der so genannte Callejón de Huaylas bezeichnet das Tal des Rio Santa in der Provinz Ancash, das von ca. 4.200m üNN bis auf unter $2.000 m$ üNN reicht. Es ist charak-terisiert durch kleinere Städte, die sich entlang des Rio Santa wie Perlen einer Kette aufreihen, wobei die Provinzhauptstadt Huaraz mit rund 130.000 Einwohner*innen mit Abstand die größte Stadt ist. Der ländliche Raum ist geprägt durch kleinbäuerliche Landwirtschaft, die im Wesentlichen auf Subsistenz und die lokalen Märkte ausgerichtet ist und neben Kartoff eln auch Mais, Weizen, Oca und Olluco produziert sowie Meerschweinchen und Weidevieh hält (Neuburger et al. 2012, Carey 2010). Während die überwiegende Mehrzahl der bäuerlichen Familien ausschließlich Regenfeldbau betreibt, können enige wenige Familien mit Anbaufl ächen in Flussnähe auf Bewässerungswasser zurückgreifen und erzielen dadurch höhere Erträge. Meist kombinieren die Haushalte den Ackerbau mit extensiver Viehzucht, indem sie die über 4.00om üNN gelegenen Talböden in den Seitentälern in einem traditionellen System der comuneros als natürliche Weiden gemeinschaftlich nutzen (Gurgiser et al. 2015). Während diese im Wesentlichen traditionell geprägte Landwirtschaft vor allem von Quechua-sprechender Bevölkerung getragen wird, haben sich in den letzten Jahrzehnten in den niedriger gelegenen Talbereichen des Rio Santa Haupttals modernisierte Formen der Bewässerungslandwirtschaft entwickelt, in denen vorwiegend mestizische Familien 
oder Unternehmer*innen insbesondere Obst und Blumen für den städtischen Markt in Lima oder für den Export produzieren (Marshall 2009, Urtega \& Boelens 2006). Darüber hinaus spielt der Megabergbau in der Region mit der Eröff - nung von zwei großen Tagebauminen eine immer größere Rolle durch die Umverteilung eines kleinen Teils der Gewinne in Form von royalties, die in der Region vor allem im Bausektor, in soziale Infrastruktur und ländliche Entwicklung investiert werden, wobei allerdings in den regionalen Tageszeitungen regelmäßig über Korruptionsfälle in den Projekten berichtet wird.

Dieser Modernisierungsimpuls, der die bestehenden Ungleichheiten in der Region weiter verstärkt, da er vor allem in neoliberalen, Technik-orientierten Bereichen zum Tragen kommt, wird überlagert von Diskursen und Aktivitäten im Kontext des globalen Klimawandels. Der seit den 1920er Jahren beobachtbare Rückzug der Gletscher der Cordillera Blanca und die damit verbundenen verheerenden Ausbrüche von Gletscherseen haben nicht nur die internationale scientifi c community nach Huaraz gebracht, sondern auch zahlreiche internationale NGOs und EZ-Organisationen, die in den letzten Jahren verstärkt in Maßnahmen zur Anpassung an den Klimawandel aktiv sind. Die dominanten Diskurse, die über globale Geldgeber*innen und Akteure in die Region getragen und dort von regionalen und lokalen Akteuren reproduziert werden, defi nieren dabei den Klimawandel im Wesentlichen als zwar vom Menschen gemachtes, aber ausschließlich in der Natur stattfi ndendes Phänomen, das entsprechend ökologische Folgen hat: erhöhte Gefahr des Ausbruchs von Gletscherseen, Rückgang der Wasserverfügbarkeit, erhöhte Temperatur- und Niederschlagsvariabilitäten, Rückgang der Biodiversität und erhöhter Schädlingsbefall in der Landwirtschaft (siehe Abb. 1). Als Hauptopfer dieser Folgen wird die traditionelle kleinbäuerliche Landwirtschaft identifi ziert, da diese - so der vorherrschende Diskursstrang - durch mangelnde fi nanzielle Ressourcen und fehlendes Wissen am verwundbarsten gegenüber diesem „,neuen“ Phänomen sei. Das so diskursiv hergestellte ökologische Problem des Klimawandels kann - so die Logik der Argumentation - nur mit (agrar-)technischen Mitteln gelöst werden. In den letzten Jahren führen deswegen die in der Region aktiven Entwicklungsagenturen verschiedene Projekte durch, die die Anpassung der andinen kleinbäuerlichen Landwirtschaft sowie die Bewusstseinsbildung der ländlichen Bevölkerung beinhalten. Neben der Einführung neuer Trockenheit- und Frostresistenterer Anbauprodukte werden wassersparende Bewässerungsanlagen angeboten und Kurse zur Umweltbildung vor allem hinsichtlich eines wassersparenden Lebenswandels durchgeführt.

Abb. 1. Dominante Diskursstränge zum Klimawandel im Callejón de Huaylas 


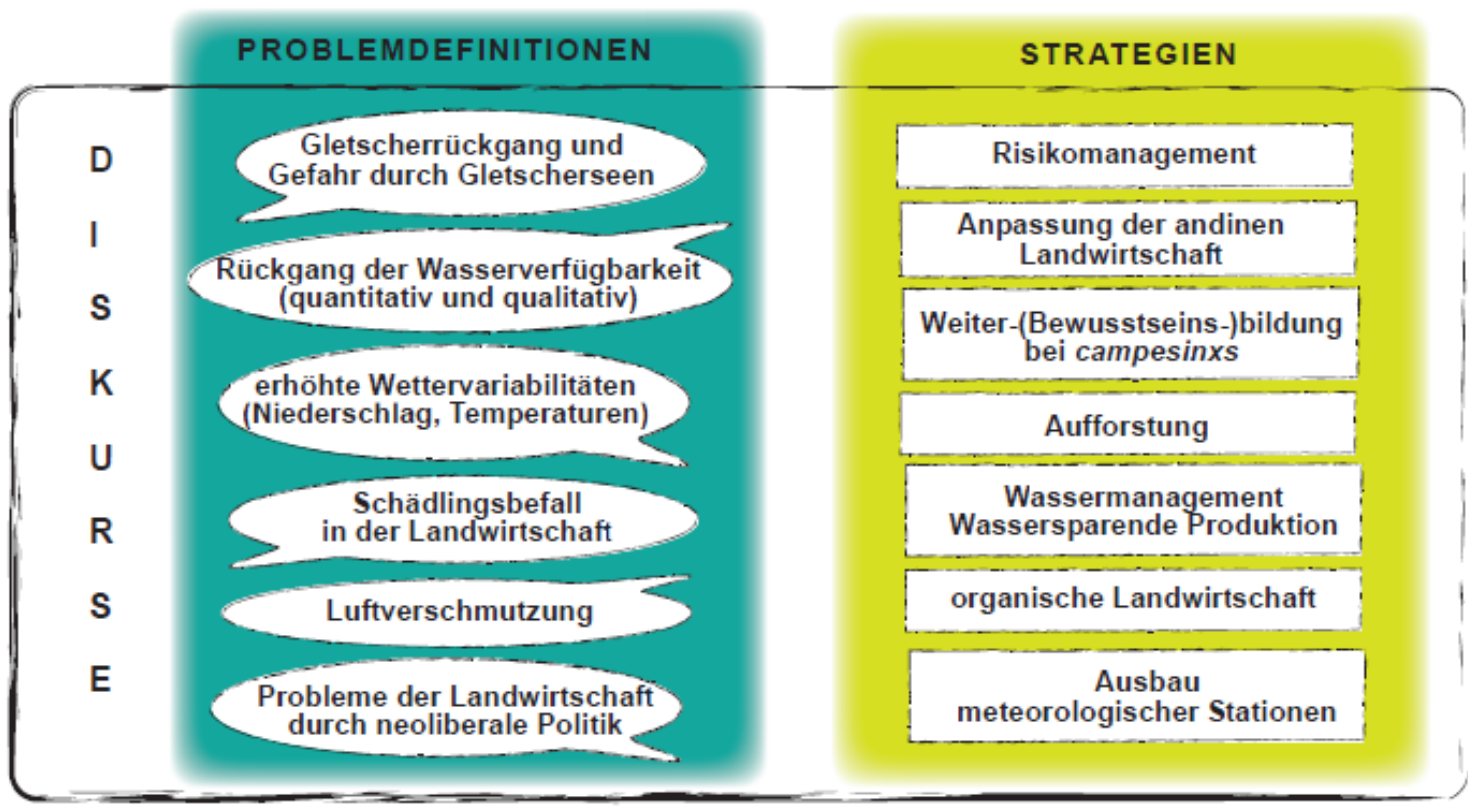

\begin{tabular}{|l|l|l|l|}
\hline \multirow{2}{*}{\begin{tabular}{l|l|l|} 
Interviews \\
2012-2014
\end{tabular}} & in Huaraz verortet & in Lima verortet & $\begin{array}{l}\text { Institution/NGO mit } \\
\text { internationalen Geldgebern }\end{array}$ \\
\cline { 2 - 4 } & FADA & SENAMHI & CARE \\
\hline & Soluciones Prácticas & CNA & AgroRural \\
\hline
\end{tabular}

Mit dieser Projektkonzeption, die sich in mehr oder weniger abgewandelter Form in der überwiegenden Mehrzahl der Entwicklungsprojekte von internationalen NGOs wie CARE, Instituto de Montaña oder der Schweizerischen EZ-Institutionen und auch bei nationalen NGOs wie Soluciones Prácticas oder Coordinadora Rural fi ndet, werden die kleinbäuerlichen Familien in eine passive und untergeordnete Rolle gedrängt. Trotz vielfach eingebauter partizipativer Methoden sollen letztlich die ländlichen Familien von den NGOs den ,richtigen“ Umgang mit Klimawandel, Umwelt und Wasser lernen. Dadurch werden die Bäuer*innen nicht nur entmündigt und zu unwissenden Hilfeempfänger*innen gemacht. $\mathrm{lhr}$ langjähriges Erfahrungswissen aus der alltäglichen landwirtschaftlichen Praxis und aus dem Umgang mit den natürlichen Variabilitäten von Temperatur und Niederschlag wird dadurch subtil negiert. Darüber hinaus zeigen aktuelle Forschungen, dass sich in den jahreszeitlichen Niederschlags werten keine deutlichen Trends der Veränderung innerhalb der letzten fünfzig Jahre nachweisen lassen (Gurgiser et al. 2015). Die Schwierigkeiten der Produktivität in der kleinbäuerlichen Landwirtschaft sind eher in sozioökonomischen und agrarpolitischen Fragen zu suchen. So legten schon in den 1980er Jahren staatliche Förderprogramme zur Modernisierung der Landwirtschaft den kleinbäuerlichen Betrieben nahe, ihre traditionellen und vielfältigen Kartoff elsorten durch „verbesserte“ Hochertragssorten auszutauschen. Diese sind jedoch gegenüber den „,normalen“ Wettervariabilitäten Trockenperioden in der Regenzeit, Frost etc. - sehr viel empfi ndlicher als die traditionellen Sorten und ihr Wachstum von kostenintensiven chemischen Düngern und dem Einstatz von Insektiziden abhängig, so dass die Produktivität vielfach sank. In den 1990er Jahren wurden außerdem durch neoliberale Politiken die Agrarmärkte geöff net. Aufgrund billiger Konkurrenzprodukte sanken daraufhin die Preise für 
lokale Agrarprodukte und die Einkünfte aus der kleinbäuerlichen Landwirtschaft mit ihnen. Schließlich bildet die Abwanderung vor allem der jungen Bevölkerung nach Huaraz oder Lima ein großes Problem, da dadurch gemeinschaftliche Arbeiten wie die Pfl ege der Bewässerungsanlagen sowie die Bewältigung von Arbeitsspitzen in der Landwirtschaft erschwert werden. Die Projekte, die die Anpassung der kleinbäuerlichen Landwirtschaft an den Klimawandel zum Ziel haben, greifen damit nicht die Ursachen des Niedergangs der Landwirtschaft an, sondern reproduzieren vielmehr hierarchische Strukturen, in denen regionale Eliten und internationale NGOs als die Träger*innen von Wissen, Kapital und Technologie auftreten und kleinbäuerliche Akteure als passive Opfer des Klimawandels in Diskurs und Praktiken hergestellt werden.

Die grundsätzliche Höherbewertung mestizischen Wissens verbunden mit der Hierarchisierung der Lebensstile, in denen städtische als den ländlichen überlegen betrachtet werden, lässt sich bis in die Kolonialzeit zurückverfolgen, in der Städte als die Machtzentren und Spanier*innen bzw. Mestiz*innen als wirtschaftliche und politische Eliten galten. Dies äußert sich noch heute sowohl in der Romantisierung des Altiplano als schöne Landschaft und Wiege der peruanischen Kultur als auch in der Herabsetzung der so genannten Sierra-Region als rückständig, traditionell und stagnierend (Trivelli et al. 2009, Murra 2002).

Die daraus resultierenden Diskriminierungsformen zeigen sich besonders deutlich im Bildungsbereich. In den ländlichen Schulen beispielsweise kommen meist Lehrer*innen zum Einsatz, die aus städtischen Kontexten kommen und ausschließ- lich Spanisch sprechen. Ihre geringe Motivation verbunden mit einer hohen Zahl an Fehlstunden hängt dabei nicht nur mit der fehlenden gesellschaftlichen Anerkennung ländlicher Lehrerjobs oder den sehr einfachen Lebensverhältnissen häufi g ohne Strom und $\mathrm{fl}$ ießend Wasser in den ländlichen Siedlungen zusammen, sondern auch mit der extrem schlechten Bezahlung, die die Lehrer*innen dazu zwingen, weitere (städtische) Jobs anzunehmen, um ihren Lebensunterhalt zu fi nanzieren. Die Kinder, die mehrheitlich aus Quechuasprechenden Haushalten kommen, leiden im Spanischsprachigen Unterricht unter Verständigungsschwierigkeiten und werden vielfach von den Lehrer*innen ob ihrer fehlenden Spanisch-Kenntnisse beschimpft, misshandelt und als weniger intelligent gegenüber den mestizischen Schüler*innen stigmatisiert. Diese Umgangsformen fi nden in den städtischen weiterführenden Schulen ihre Fortsetzung in der kontinuierlichen Diskriminierung und Herabwürdigung von Kindern und Jugendlichen aus ländlichen Regionen (Morales \& Singh 2015, Pasquier-Doumer \& Risso Brandon 2015).

Wie wirkmächtig diese hegemonialen Lebensstile gleichermaßen bei Schüler*innen und Lehrer*innen sind, zeigen Befragungen mit Kindern in den Schulen von Ichoca und Chontayoc, zwei ländlichen Siedlungen im Callejón de Huaylas. Auf die Frage nach ihren Berufswünschen antworteten alle Kinder mit gut qualifi zierten städtischen Berufen wie Ärzt*in, Rechtsanwält*in, Bankangestellte*r, Bergbauingenieur*in etc., was einer Negierung der eigenen ländlich-bäuerlichen Herkunft gleichkommt, während der Lehrer bei der Erklärung der Aufgabe ausschließlich niedrig qualifi - zierte Berufe wie Verkäufer*in, Fahrer*in, Hausangestellte* $r$ etc. nannte, weil - so die vorherrschende Meinung akademische Berufe für Kinder aus ländlichen Kontexten unzugänglich sind (Singer 
\& Villari 2016). Damit werden soziale und ethnische Ungleichheiten reproduziert und verstärkt. Auch die gesetzliche Verankerung und Einführung von bilingualem Unterricht hat an dieser Grundsituation nichts geändert, da die entsprechenden schulischen Reformen nur sehr zögerlich umgesetzt werden (Grompone 2005, Lozano Vallejo 2000).

In diesem Kontext kann das allmähliche Verschwinden der Sprache Quechua bzw. der Verlust ihrer internen Vielfalt und Komplexität verstanden werden als andauernder Verdrängungsprozess der ländlichen Lebensentwürfe aus der gesellschaftlichen Anerkennung. Dieses Phänomen ist in Ancash besonders deutlich zu sehen, da der regional-spezifi sche Quechua-Dialekt schon seit der Kolonialzeit als dem „Hoch-Quechua“ aus Cuzco untergeordnet gesehen wird. Im QuechuaSpanisch zweisprachigen Umfeld des Callejón de Huaylas verliert damit Quechua immer mehr an Bedeutung, was nicht nur einer Reduzierung sprachlich-kultureller Diversität gleichkommt, sondern was auch mit dem Verlust des in der Sprache eingewobenen Wissens über Natur, Religion und Kosmovision verbunden ist.

\section{Privilegierungsmechanismen in Pozuzo}

Diesen vielfältigen Diskriminierungsformen Quechua-sprechender Bevölkerung, denen auch afro-peruanische Gruppen ausgesetzt sind, stehen umfangreiche Privilegierungen von mestizischen und europäisch-stämmigen Bevölkerungsgruppen gegenüber. Die Mechanismen und postkolonialen Verwobenheiten solcher Privilegierungen werden im Folgenden am Beispiel von Pozuzo, einem Munizip in der Selva Central, dargestellt. Das Munizip defi niert seinen Ursprung im 19. Jahrhundert, als sich im Jahr 1859 im Rahmen eines peruanischen Ansiedlungsprojektes rund 170 deutschsprachige, katholische Kolonist*innen, etwa 120 Tiroler*innen sowie 50 Rheinländer*innen, am Zusammenfl uss von Rio Santa Cruz und Rio Huancabamba niederließen. Die dort bereits ansässige indigene Gruppe der Yanesha zog sich in andere Gebiete zurück (Santos-Granero 2004). Es ist nicht bekannt, ob daraus Konfl ikte entstanden. Die offi zielle Geschichtsschreibung von Pozuzo spricht davon, dass die Einwander*innen und die indigenen Gruppen friedlich nebeneinander lebten (Laura Contreras 2007, Santos-Granero \& Barclay 1998, Abendroth 1870).

Die Kolonie war bereits in ihren Anfängen ein Zuwanderungsgebiet der indigenen und mestizischen Bevölkerung, so dass dadurch schon Anfang des 20. Jahrhunderts die deutschstämmigen Gruppen in die Minderheit kamen. Der darauf folgende Anstieg der Einwohner*innen über die Jahrzehnte hinweg führte einerseits dazu, dass mehrere Tochtersiedlungen in der Umgebung gegründet wurden. Andererseits wurden durch Heirats- und Familiengründungen die ethnischen Trennungen zunehmend aufgehoben. Die Umstellung der anfänglichen Subsistenzwirtschaft auf eine intensive marktorientierte Viehzucht, aber auch Intensivierungen im Kaff eeanbau sowie die mit dem plötzlichen Ansteigen des Cocapreises verbundene Ausweitung der Cocaproduktion verbreiterten die wirtschaftliche Basis. Mit der wachsenden Prosperität - mit einer etwa zehnjährigen Zäsur Ende der 1980er bis Ende der 1990er Jahre, in der die terroristische Organisation Sendero Luminoso in die Region vordrang - gingen verstärkte Zuzüge 
weiterer Bevölkerungsgruppen aus der nahen und weiteren Umgebung einher (Th imm 2001, Wencelides 1986).

Die dargestellten Entwicklungen haben bis zum heutigen Tag dazu geführt, dass die ethnische Diversität im Distrikt Pozuzo kontinuierlich angestiegen ist und die Trennung zwischen den einzelnen ethnischen Gruppen immer schwieriger wird. Als eine der Folgen dieser soziokulturellen Prozesse verschwinden Tiroler Sprache und Bräuche immer mehr aus dem Pozuziner Alltag. Insbesondere die Assimilation an die Staatssprache hat sich zweifellos auch auf die ethnische Identifi kation niedergeschlagen (Steinicke \& Vavti 2006). Trotz der ethnischen Durchmischung stehen die meisten Familien mit deutschstämmigen Wurzeln, ob sie nun den Tiroler Dialekt sprechen bzw. Tiroler Bräuche praktizieren oder nicht, dem Erhalt der alten Gebräuche positiv gegenüber. Es geht um die Selbstidentifi kation, eine Pozuzina bzw. ein Pozuzino und damit „mehr als nur ein*e Peruaner*in zu sein. Dieses „Mehr“ drückt sich etwa in „Rückbesinnungen“ - wie es von den Protagonist*innen genannt wird - auf „alte“ "Traditionen aus, deren tatsächlicher Ursprung jedoch fragwürdig bleibt. Entsprechend grenzen sich die deutschstämmigen Familien sehr stark gegenüber Familien ab, die in ihrer Wahrnehmung eine andere ethnische Zugehörigkeit haben.

Das Aufl eben von Tiroler Traditionen wird seit den 1980er Jahren stark von Fördervereinen aus Tirol forciert. Zu Beginn der 1970er Jahre bereiste der Innsbrucker Ethnologe Karl Ilg im Auftrag der Tiroler Landesregierung die ehemaligen Tiroler Siedlungen in Südamerika. Seine Aufgabe war es, dort den „Stand der Tiroler Kultur" zu erkunden und - sollte sie „bedroht" sein - geeignete Maßnahmen für ihren Erhalt vorzuschlagen. Wie in den anderen Siedlungen suchte er auch in Pozuzo Personen auf, die er dazu animierte, Tiroler Sprache und Bräuche aufrechtzuerhalten und gegen die „Bedrohung“ durch die indigene Kultur zu verteidigen. Diese Idee der unbedingten Bewahrung der Tiroler Kultur schlug sich 1962 in der Gründung des Vereins „Freundeskreis für Pozuzo“ in Tirol nieder, der allerdings 1980 wieder aufgelöst wurde. Wenige Jahre später - im Jahr 1983 wurde dieser Verein unter gleichem Namen neu gegründet. Finanziert durch Spenden und Zuschüsse der Tiroler Landesregierung widmete er sich zunächst der Verbesserung der Infrastruktur im Distrikt Pozuzo. Schulen, Straßen, Wege und Brücken wurden gebaut. Für den Bau des Krankenhauses wurde 1997 eigens der Verein „Gesundheit für Pozuzo“ gegründet. Darüber hinaus fi nanzierte der Verein Deutschkurse an der örtlichen Schule, die zunächst Tiroler Lehrerinnen (sic!) leiteten, heute jedoch von Pozuzinerinnen (sic!) selbst durchgeführt zum festen Bestandteil des Schulunterrichts zählen und seit 2016 von der Gemeindeverwaltung Pozuzo getragen werden. In jüngster Zeit bekommen außerdem einzelne Jugendliche aus Pozuzo die Möglichkeit, in Tirol über mehrere Monate oder Jahre hinweg spezielle Ausbildungsgänge - vor allem im Tourismusbereich, aber auch in handwerklichen Fertigkeiten etc. - zu besuchen oder dort zu arbeiten. Im Jahr 2009 anlässlich des 150-jährigen Jubiläums spendeten außerdem Tiroler Handwerksbetriebe gebrauchte Maschinen und Gerätschaften, um das Kleingewerbe in Pozuzo zu fördern. All diese Maßnahmen - sieht man vom Krankenhausbau ab - kamen vorwiegend denjenigen Familien zugute, die sich zu den deutschstämmigen Siedler*innen zählen und von den Tiroler Vereinen auch als solche akzeptiert werden. Straßen und Wege wurden vor allem zu denjenigen 
caseríos gebaut, die von Tiroler Familien bewohnt sind. Ausbildungen in Tirol wurden und werden den Jugendlichen aus eben diesen Familien angeboten. Die Maschinen-Spenden aus Tirol nützten nur den deutschstämmigen Familien, da die als indigen bezeichnete Bevölkerung Pozuzos vorwiegend in der kleinbäuerlichen Landwirtschaft tätig ist.

Im Jahr 2005 schließlich wurde in Tirol der Verein „Pro Kultur Pozuzo“ gegründet, der sich besonders der Förderung von Tiroler Kultur und Brauchtum verschrieben hat. Es handelt sich dabei um eine Ausgründung aus dem Verein „Freundeskreis für Pozuzo“. Die Protagonist*innen des neuen Vereins sahen in der bisherigen Arbeit des älteren Vereins die Kulturförderung als vernachlässigt an und konzentrieren ihre Aktivitäten dementsprechend auf diesen Bereich. Sie unterstützten den Bau des Kulturhauses „Casa de Cultura“ und setzen sich seit seiner Fertigstellung für die „,angemessene“ Nutzung ein. Von zentraler Bedeutung ist aus Sicht des Vereins dabei der „Erhalt der Tiroler Kultur“. Darunter versteht er die Förderung von Liedern und Tänzen, die in der Regel in Form von CDs und DVDs nach Pozuzo transportiert und dort in regelmäßigen Übungsabenden einstudiert werden. Außerdem werden Trachten - Dirndl und Lederhosen - nach Pozuzo geschickt, um gewissermaßen auch die kulturellen Artefakte anzupassen.

Die Pozuziner Bevölkerung nimmt diese Sachspenden zwar gerne an. Allerdings bilden sie nicht die Fortführung einer Tradition, da weder Trachtentragen noch aktuelle Tiroler Tänze und Lieder jemals von den ursprünglichen Tiroler Siedlerfamilien in Pozuzo praktiziert worden waren. Manchen deutschstämmigen Pozuziner*innen ist diese ,kulturelle Bevormundung“ auch sichtlich fremd. Gleichzeitig nutzen sie diese als willkommenen Identifi kationsanker und als Brücke zur „Heimat“ Tirol. Selbst junge Mestiz*innen, die nicht von den deutschstämmigen Kolonist*innen abstammen, beteiligen sich an der entsprechenden Vereinsarbeit nicht zuletzt, um auf diesem Wege der dominanten Tiroler Kultur - gleichbedeutend mit der Kultur der wirtschaftlichen und politischen Elite - ebenfalls anzugehören. Gleichzeitig ergeben sich daraus neue Verdienstmöglichkeiten und Privilegien, da in der touristischen Saison von den örtlichen Restaurants so genannte „Tiroler Abende" veranstaltet werden, bei denen die Teilnehmer*innen der Tanzkurse gegen Bezahlung auftreten. Im Mittelpunkt stehen damit nicht der Erhalt der Tiroler Kultur an sich, sondern das Symbol für die Identität der österreichisch-deutschen Kolonie, nunmehr des peruanischen Pozuzo, und die damit verbundenen Einkommenschancen. Dies könnte im weitesten Sinne auch als ein Ausdruck der „symbolischen Ethnizität" verstanden werden, wobei die alten Gebräuche eine Art Hülle darstellen, deren Inhalt - beispielsweise der des Tiroler Dialekts - allerdings mittlerweile zum allergrößten Teil schon verloren gegangen ist (Gans 1979).

Die Bereitwilligkeit und die Eigeninitiativen der deutschstämmigen Bevölkerung in der Forcierung der Tiroler kulturellen Praktiken hängen auch damit zusammen, dass diese touristisch sehr gut inwertzusetzen sind. Seit dem weitgehenden Verschwinden des Sendero Luminoso aus der Region wächst der Tourismus kontinuierlich an. Waren im Jahr 1990 noch 95 \% der Erwerbsbevölkerung von Pozuzo in der Landwirtschaft tätig, so fi el dieser Wert bis 2007 auf 64 \% (von insgesamt 2.456 Personen). Gleichzeitig stieg der Anteil, der im tertiären Wirtschaftssektor beschäftigt ist, von 4 auf $16 \%$ an, von denen ein knappes Viertel im Tourismus tätig sein dürfte (Gobernación Pozuzo 2008, Municipalidad Distrital de 
Pozuzo 2007, Comite de Desarrollo Comunal 1990, INEI 2008). Im Vergleich zum Agrarsektor erscheint diese Zahl zunächst unbedeutend. Aus dem Stellenwert, den der Tourismus in den aktuellen Entwicklungsplänen einnimmt, lässt sich jedoch ableiten, dass in diesen Wirtschaftssektor große Hoff nungen für die Zukunft gesetzt werden. Die seit 1994 konstant höher werdende Zahl an Gästeankünften, der Ausbau der touristischen Infrastruktur sowie die Anstrengungen in der Ausweitung des Freizeitangebots weisen außerdem auf den tatsächlichen Bedeutungszuwachs dieser Branche im Distrikt Pozuzo hin. Impulsgeber für die Tourismusentwicklung waren deutschstämmige Pozuzinxs. Mit dem Slogan „única colonia austro-alemana del mundo" - einzige österreichisch-deutsche Kolonie der Welt - ist es der alten Kolonie gelungen, den Namen „Pozuzo“ in Peru bekannt zu machen und gleichzeitig auf seine besonderen natur- und kulturräumlichen Gegebenheiten hinzuweisen.

Dazu kommt, dass in Lima, dem Hauptherkunftsgebiet der Tourist*innen, die Zeit des tagsüber persistenten Küstennebels („invierno“) mit der sonnigen Trockenzeit in Pozuzo (,verano“) zusammenfällt. Aus diesem Grund genießen viele Limeñxs zwischen Mai und September das angenehme tropische Klima von Pozuzo. In den Seitentälern bieten die zahlreichen, durch Wege und Pfade erschlossenen caseríos lohnende Wanderungsziele. Dabei besteht die touristische Attraktion darin, dass sich Pozuzo von den übrigen peruanischen Siedlungen markant durch seine noch immer vorhandenen Eigenarten in Baustil und -material unterscheidet, außerhalb der Siedlungen, aber auch durch die stark an die Tiroler Kulturlandschaft erinnernden Rodungsinseln und Flurformen. Zudem unterstützt der im Jahr 1986 etwas oberhalb von Pozuzo ausgewiesene Nationalpark Yanachaga Chemillen das Pozuziner Konzept des Ökotourismus.

Die Distriktführung hat in den 1990er Jahren die touristische Dimension der siedlungsgeographischen und ethno-kulturellen Besonderheit erkannt. Ein erster Schritt war 1995 die Schaff ung des Día del Colono (Tag des Kolonisten), der auf den 25. Juli festgelegt wurde und an den Gründungstag der Kolonie im Jahr 1859 erinnern soll. Es folgten weitere bedeutende Initiativen, wie die Tourismuswoche (Semana Turí- stica de Pozuzo) seit Juli 1997 oder das seit 1998 jährlich im Oktober stattfindende

Abb. 2: Touristische Einrichtungen in den Hauptsiedlungen in Pozuzo und Prusia 


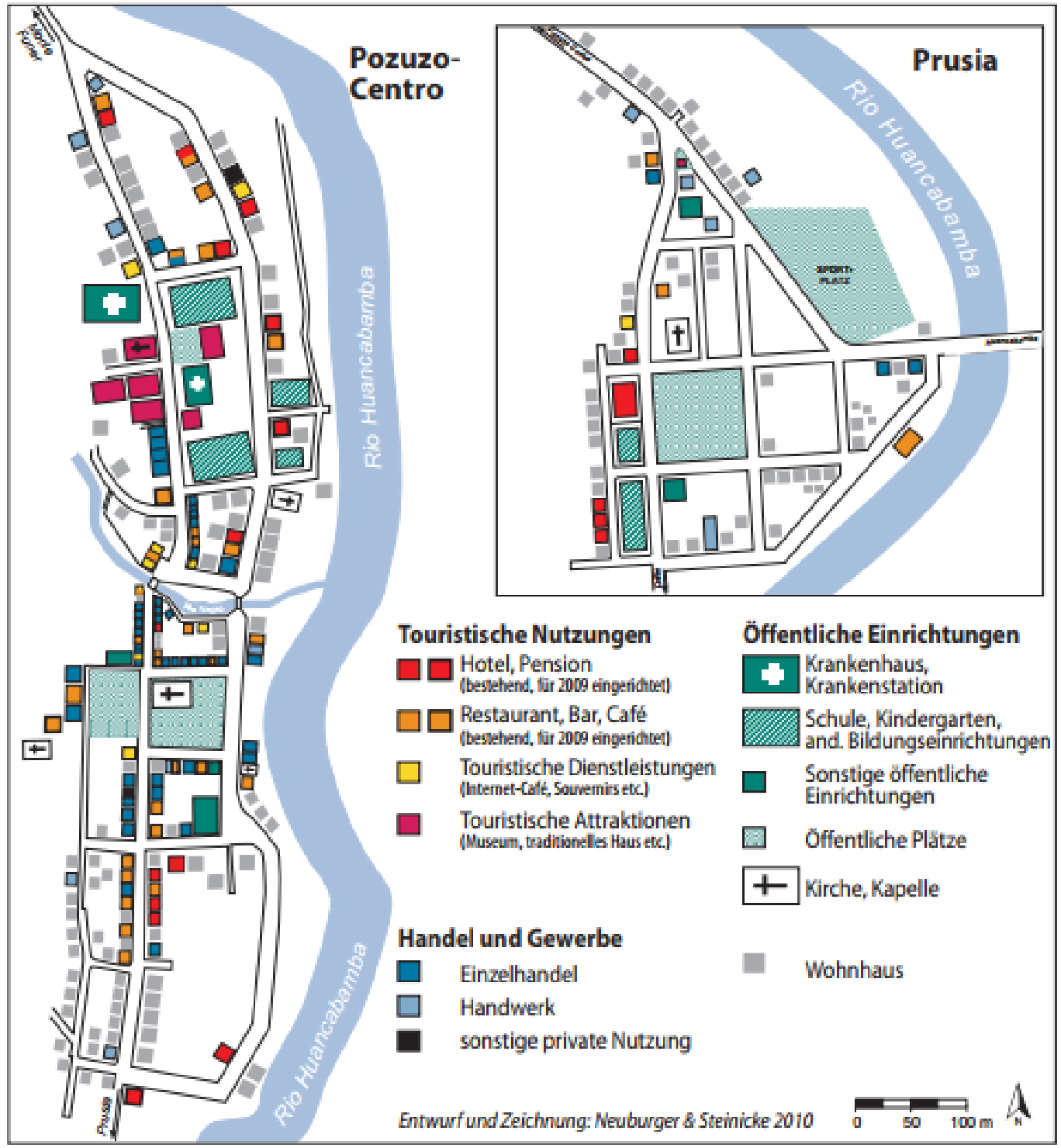

Festival Ganadero y Ecoturismo (Fest der Viehzüchter und des Ökotourismus). Alle deutschstämmigen wie indigenen Bürgermeister (sic!) waren bemüht, in den Entwicklungsplänen von Pozuzo dem Kulturtourismus sowie dem Ökotourismus einen hohen Stellenwert beizumessen. Entsprechend sind in den letzten Jahren auch die auf den Tourismus ausgerichteten Betriebe - Hotels, Pensionen, Restaurants, Bars etc. - sehr stark erweitert worden (siehe Abb. 2). Vor allem anlässlich des 150-jährigen Jubilä- ums wurden zahlreiche Pensionen und Restaurants neu eröff net, die allerdings kurz nach dem Event im Juli 2009 ihre Pforten aufgrund mangelnder Nachfrage wieder schließen mussten.

Das wirtschaftliche Potenzial des Tourismus bleibt dadurch mehr als fraglich. Die gastronomischen Betriebe, Pensionen und Gästehäuser sind in den Monaten November bis Mai weitgehend geschlossen. Interviews bestätigen den Eindruck, dass der Lebensunterhalt der Familien nur durch entsprechende zusätzliche Beschäftigungen und Einkünfte aufrechterhalten werden kann. Darüber hinaus verschließt gerade die Konzentration der Tourismusförderung auf die Tiroler Kultur diesen Wirtschaftssektor für Bevölkerungsgruppen, die nicht zu den deutschstämmigen gezählt werden. Amazonisch-indigene und Quechua-sprechende 
Bevölkerung fi nden darin maximal als schlecht bezahlte Arbeitskräfte einen Job. Damit verstärkt der Ausbau des Tourismus die sozioökonomische Polarisierung entlang den (re-)produzierten Trennlinien zwischen den verschiedenen Ethnien in der Pozuziner Bevölkerung, denn die Nachkommen der Tiroler Kolonist*innen bilden seit jeher die obere soziale Schicht in Pozuzo und sind dort stets an den wirtschaftlichen sowie politischen Entscheidungsprozessen maßgeblich beteiligt gewesen.

In der Landwirtschaft bilden die deutschstämmigen Familien aufgrund der Landzuteilung bei der Ansiedlung in Pozuzo und der meist informellen Landnahme in den Expansionsgebieten als Landeigentümer*innen und Viehzüchter*innen die Oberschicht, während amazonisch-indigene und Quechua-sprechende Bevölkerung vorwiegend als Angestellte und Tagelöhner*innen in den Viehzuchtbetrieben arbeiten. Diese wirtschaftliche Basis erlaubte es den Familien mit Tiroler Herkunft, in den Tourismus zu investieren und teilweise stattliche Pensionen und Gaststätten aufzubauen. Auch in diesem Sektor werden die nicht als deutschstämmig Akzeptierten auf die Position der abhängigen - meist schlecht bezahlten und angelernten - Arbeitsplätze verwiesen. Dies ist darauf zurückzuführen, dass den zuletzt Genannten das notwendige Kapital fehlt. Gleichzeitig entsprechen auch Kleidung, Lebensweise und körperliche Statur nicht der von den Tourist*innen nachgefragten „Tiroler Kultur“, was wiederum als Reproduktion der neokolonialen und rassistischen Gesellschaftsordnung verstanden werden kann. Entsprechend ihrer wirtschaftlichen Position bekleideten die deutschstämmigen Siedler*innen bislang auch die politischen Leitungsfunktionen im Distrikt. Da die Tirol-stämmigen Bürgermeister (sic!) politische Prioritäten setzten, die vorwiegend ihrer eigenen Ethnie zugutekamen, verstärkten politische Exklusionsprozesse die ohnehin schon wirtschaftlich bestehenden Disparitäten. So ist es zu verstehen, dass in soziolinguistischer Hinsicht noch immer das Tirolerische, welches heute nur noch ein Bruchteil der Bevölkerung täglich verwendet, an oberster Stelle gesellschaftlicher Anerkennung steht, gefolgt vom Castellano, während die Verwendung von Quechua als rückständig gilt. Erst langsam löst sich die Dominanz des Deutschstämmigen auf, was sich in einem zumindest vorübergehenden Wechsel an der politischen Führungsspitze niedergeschlagen hat: Für die Legislaturperioden 2007-2010 und 2015-2018 wurden mestizische - von den deutschstämmigen als „indigen“ bezeichnete - Bürgermeister gewählt.

Die Aktivitäten der Tiroler Fördervereine unterstützen mit Infrastrukturmaßnahmen und Kulturförderung die landwirtschaftliche und touristische Dynamik in Pozuzo. Dies wird von allen Bewohner*innen keineswegs als aufgebürdet empfunden, sondern ist im Gegenteil in hohem Maße erwünscht und verinnerlicht. Die kulturellen Maßnahmen bilden auch Anknüpfungspunkte für touristische Managementstrategien: Pozuzo hat in Peru als österreichisch-deutsche Kolonie einen klingenden Namen. Auf der anderen Seite schaff en die genannten Aktivitäten gleichzeitig eine wachsende kulturelle Distanz zwischen denjenigen, die sich als deutschstämmig fühlen, und jenen, die nicht dazugehören wollen oder können. Exklusionsprozesse werden dadurch sozial, politisch und wirtschaftlich verschärft und eine Kommunikation zwischen den (konstruierten) ethnischen Bevölkerungsgruppen erschwert. 
Diese soziokulturellen Dynamiken schreiben somit koloniale Hierarchien bis heute fort. Auch wenn die österreichischen und deutschen Familien aus den ärmsten Schichten ihrer Heimatländer kamen, erfuhren sie als europäische Siedler*innen in Peru eine bessere Behandlung als die amazonisch-indigene oder die Quechua-sprechende Bevölkerung. Sie erhielten mit ihrer Ankunft in der Selva Central vom peruanischen Staat das Recht, sich Land anzueignen und - als Basis ihres heutigen wirtschaftlichen Erfolges - entsprechende Landtitel kostenfrei zu erwerben, während die Nutzungsrechte der ansässigen Yanesha-Gruppen ignoriert wurden. Diese sowie die später zugewanderten Quechua-sprachigen Gruppen hatten ihrerseits nur die Möglichkeit, Land käufl ich zu erwerben oder es „illegal“ zu besetzen. Da ein Landkauf aufgrund des Kapitalmangels nur selten möglich war, geschah letzteres in den $1980 e r$ und 1990er Jahren sehr häufi g, als diese Gruppen in Pozuzo Zufl ucht vor den Attacken des Sendero Luminoso suchten. Damit wurde von vorneherein eine Hierarchie geschaff en, die bereits in den kolonialen Beziehungen zwischen Europa und Lateinamerika angelegt war.

\section{Fazit}

Sowohl die beiden Beispiele als auch die allgemein dargestellten Ungleichheiten in der peruanischen Bevölkerung sind in ihrer heutigen Konstitution als ein Gefl echt von Hegemonie und Machtbeziehungen in ihrem historischen Gewordensein zu verstehen. Die bereits in der inkaischen Gesellschaft angelegten Hierarchien wurden in der Kolonialzeit nicht gebrochen, sondern von der spanischen Krone reproduziert und mit eigenen Machtstrukturen überlagert (Sobrevilla 2001). Die Systeme der Feudal-gesellschaft mit Leibeigenschaft und Sklaverei wirken bis heute nach. Die hierarchischen Beziehungen zwischen den unterschiedlichen ethno-sozialen Gruppen sind bis heute durch die verschiedensten Formen von Diskriminierung und Rassismen wirkmächtig, wenn es um den Zugang zu Land und Einkommen, Bildung und gesellschaftliche Anerkennung geht (Henk 2010, Lozano Vallejo 2000, Golash-Boza 2011).

Dennoch: Trotz der scheinbar über Jahrhunderte verfestigten Strukturen erreichen politische Initiativen und soziale Bewegungen immer wieder gesellschaftliche Veränderungen, die in dynamische Prozesse münden. Neben der Befreiungstheologie, die die Koalition der Kirche mit den politischen Eliten aufkündigte, sind es Widerstandsbewegungen der afro-peruanischen Bevölkerung ebenso wie Frauenorganisationen und indigene Gruppen, die häufig in Verbindung mit international aktiven NGOs erfolgreich ihre Rechte einfordern. Das seit 1998 verfassungsrechtlich verankerte Bekenntnis des peruanischen Staates zur Multiethnizität und Plurikulturalität verdeutlicht diese Errungenschaften und eröff net Chancen für mehr Gleichberechtigung und soziale Gerechtigkeit. 


\section{LITERATUR}

ABENDROTH, R. (1870): Die Colonie am Pozuzu in ihren physischen, ökonomischen und politischen Verhältnissen. Jahresberichte des Vereins für Erdkunde zu Dresden, 6/7: $1-58$.

ACOSTA, P. \& CALDERÓN, C. \& FAJNZYLBER, P. \& Lopez, H. (2006): Remittances and Development in Latin America. Th e World Economy, 29 (7): 957-988. doi: 10.1111/j.1467-9701.2006.00831.x

ACOSTA, P. \& CALDERÓN, C. \& FAJNZYLBER, P. \& Lopez, H. (2008): What is the Impact of International Remittances on Poverty and Inequality in Latin America? World Development, 36 (1): 89-114. doi:10.1016/j.worlddev.2007.02.016

BOLETÍN de Arqueología PUCP (2002, 2003, 2004): Identidad y transformación en el Tawantinsuyu $y$ en los Andes coloniales. Perspectivas arqueológicas y etnohistóricas. 3 Teile. Lima (PUCP).

BORSDORF, A. (1986): Dalmatinische Einwanderer am Rande der Welt. Umfang und Motive der südslawischen Auswanderung nach Südpatagonien. Österreichische Osthefte, 28 (4): 426-439.

BORSDORF, A. (1995): Deutsche und Österreicher in Chile. Einwanderung und Eingliederung. Innsbrucker Jahresbericht 1993-94. Innsbruck, 74-86.

BORSDORF, A. (1997): Austria in Amerika. Zur Kulturlandschafts- und Stadtentwicklung im frühen kolonialen Lateinamerika. Österreich in Geschichte und Literatur mit Geographie, 41 (1): 45-60.

BORSDORF, A. (2007): 150 Jahre Tiroler in Chile: Die Zillertaler am Llanquihuesee. Tiroler Heimatblätter, 82: 98-104.

BORSDORF, A. \& STADEL, C. (2013): Die Anden: Ein geographisches Porträt. Berlin.

BREA, J. A. (2003): Population dynamics in Latin America. Population Bulletin, 58 (1): $21-25$.

CAREY, M. (2010): In the Shadow of Melting Glaciers: Climate Change and Andean Society. Oxford.

COMITE desarrollo comunal (1990): Diagnóstico situacional de Pozuzo 1990. Pozuzo.

FINER, M. \& JENKINS, C. N. \& PIMM, S. L. \& KEANE, B. \& ROSS, C. (2008): Oil and Gas Projects in the Western Amazon: Th reats to Wilderness, Biodiversity, and Indiginous Peoples. PLoS ONE, 3 (8): e2932. doi:10.1371/journal.pone.0002932. 
FUENZALIDA, F. (2009): La agonía del Estado-Nación. Poder, raza y etnia en el Peru contemporáneo. Lima.

GANS, H. (1979) Symbolic ethnicity: Th e future of ethnic groups and cultures in America. - In: Gans, H. \& Glazer, N. \& Gusfi eld, J. \& Jencks, C. (Hrsg.): On the making of Americans. Essays in honor of David Riesman. Pennsylvania, 193-220.

GOBERNACIÓN POZUZO (2008): Diagnóstico Situacional del Distrito de Pozuzo 2008. Pozuzo.

GOLASH-BOZA, T. M. (2011): Yo Soy Negro: Blackness in Peru. Gainesville.

GOLTE, J. (2005): La construcción de la naturaleza en el mundo prehispánico andino, su continuación en el mundo colonial y en la época moderna. Revista de Antropología (UNMSM), 3 (3): 13-62.

GROMPONE, R. (2005): Modernidad, identidades políticas y representación: cuatro décadas y un desenlace abierto. - In: IEP (Hrsg.): El estado está de vuelta: desigualdad, diversidad y democracia. Perú Problema, 30. Lima, 187-273.

GRIFFI THS, N. (1996): Th e cross and the serpent: religious repression and resurgence in colonial Peru. Norman, Oklahoma, London.

GURGISER, W. \& JUEN, I. SINGER, K. \& NEUBURGER, M. \& SCHAUWECKER, S. \& HOFER, M. \& KASER, G. (2015): Comparing peasants' perceptions of precipitation change with precipitation records in the tropical Callejón de Huaylas, Peru. Earth System Dynamics Discussions, 6: 1-33. DOI: 10.5194/ esdd-6-1-2015

HENK, E. (2010): Von der Scham einheimisch zu sein: Ursachen und Entwicklung des Rassismus in Peru. Gießen.

HENSEL, S. (2013): Rassismus. - In: HENSEL, S. \& POTTHAST, B. (Hrsg.): Das Lateinamerika Lexikon. Wuppertal, 277-279.

HENSEL, S. \& POTTHAST, B. (Hrsg.) (2013): Das Lateinamerika Lexikon. Wuppertal.

HERRERA, A. (2003): La Serpiente de Oro y los inkas: la ocupación inka en el alto Marañón y el puerto balsero de Pogtán. Boletín de Arqueología PUCP, 7: 189-215.

HERRERA, A. (2007): Social landscapes and community identity: the social organization of space in the north-central Andes. - In: Kohring, S. \& Wynne-Jones, S. (Hrsg.): Socialising complexity: Approaches and interaction in the archaeological record. Oxford, 161-185.

HUBER, A. \& STEINHAUF, P. (1997): Soziale Netzwerke und ökonomische Entwicklung in Peru. Die neuen Protagonisten. Anthropos, 92: 535-549. 
INEI - Instituto Nacional de Estadística e Informática (2007): Censos Nacionales de Población y Vivienda. Lima.

INEI - Instituto Nacional de Estadística e Informática (2008): Censos Nacionales 2007, Resultados Defi nitivos, Departamento de Pasco, 21 de Octubre de 2007, Tomo I y Tomo II, Lima.

KLARÉN, P. F. (2004): Nación y Sociedad en la Historia del Perú. Lima.

LAURA CONTRERAS, W. (2007): Pozuzo a través de su historia. Pozuzo. (http://www.espejodelperu.com.pe/ca/per-ale/Huellas/Pozuzo-Historia.pdf).

LOZANO VALLEJO, R. (2000): Análisis de la problemática de la educación bilingüe en la Amazonía peruana. Documento de Trabajo, 4. Cusco.

MARSHALL, A. (2009): S’approprier le désert. Agriculture mondialisée et dynamiques socio-environnementales sur la peímont cótier du Pérou. La cas des oasis de Virú et d'Ica-Villacuri. Paris.

MORALES, A. \& SINGH, P. (2015): Th e eff ects of child physical maltreatment on nutritional outcomes: evidence from Peru. Journal of Development Studies, 51 (7): 826-850. DOI: 10.1080/00220388.2015.1034110.

MUNICIPALIDAD Distrital de Pozuzo (2007): Plan de Desarrollo Concertado 20072010. Pozuzo.

MURRA, J. V. (2002): El mundo andino. Población, medio ambiente y economía. Historia Andina, 24. Lima.

NEUBURGER, M. \& STEINICKE, E. (2013): (Re-)Valorización de la Identidad AustroAlemana y Confl ictos Político-Culturales en Pozuzo (Pasco, Perú). Potencialidades y Límites de la Diversidad Cultural. - In: Salas Quintanal, H. \& Serra Puche, M.C. \& González de la Fuente, Í. (Hrsg.): Identidad y Patrimonio Cultural en América Latina: la Diversidad en el Mundo Globalizado. México (UNAM, IIA), 453-488

NEUBURGER, M. \& KASER, G. \& BREGULLA, D. \& GURGISER, W. (2012): Peasant vulnerbaility, glacier mass decline and water availability: Case study concerning the eff ects of changing runoff regimes in the Cordillera Blanca, Peru. - In: Sandner Le Gall, V. \& Wehrhahn, R. (Hrsg.): Geographies of Inequality in Latin America. Kieler Geographische Schriften, 123. Kiel, 77-93.

NEWSON, L. A. (2006): Th e Demographic Impact of Colonisation. - In: Bulmer-Th omas, V. \& Coatsworth, J. H. \& Cortés Conde, R. (Hrsg.): Th e Cambridge Economic History of Latin America. Th e Colonial Era and the Short Nineteenth Century. Cambridge. 
ONKEN, H. (2013): Peru. - In: HENSEL, S. \& POTTHAST, B. (Hrsg.): Das Lateinamerika Lexikon. Wuppertal, 265-272.

PASQUIER-DOUMER, L. \& RISSO BRANDON, F. (2015): Aspiration failure: a poverty trap for indigenous children in Peru? World Development, 72: 208-223. DOI: 10.1016/j.worlddev.2015.03.001

PERZ, S. G. \& ARAMBURú, C. \& BREMNER, J. (2005): Population, Land Use and Deforestation in the Pan Amazon Basin: A Comparison of Brazil, Bolivia, Colombia, Ecuador, Perú and Venezuela. Environment, Development and Sustainability, 7: 2349. DOI 10.1007/s10668-003-6977-9

QUIROZ CHUECA, F. (2007): De la colonia a la republica independiente. Historia del Perú. Barcelona, 666-830.

RODRIGUÉZ VIGNOLI, J. (2004): Migración Interna en América Latina y el Caribe: Estudio Regional del Período 1980-2000. Serie Población y Desarrollo, 50. Santiago de Chile (CEPAL).

RÖSING, I. (2001): Die heidnischen Katholiken und das Vaterunser im Rückwärtsgang: zum Verhältnis von Christentum und Andenreligion. Heidelberg

SANTOS-GRANERO, F. \& BARCLAY, F. (1998): Selva Central: history, economy, and land use in Peruvian Amazonia. Washington D.C

SILVA, S.A. da (2003): Virgem / Mãe / Terra. Festas e Tradições Bolivianas na Metrópole. São Paulo.

SINGER, K. \& VILLARI, C. (2016): Uniendo burbujas de pensamiento. Búsqueda de diálogo interdisciplinarion entre una lingüista y und geógrafa sobre el trabajo con niños en una región de quechuahablantes. Indiana, 33 (1) - in Druck

SOBREVILLA, D. (2001): Zur Entwicklung und Lage der Kulturen in Peru. - In: Sevilla, R. \& Sobrevilla, D. (Hrsg.): Peru - Land des Versprechens? Bad Honnef, 76-109.

SPEDDING, A. (2008): Religión en los Andes: extirpación de idolatrías y modernidad de la fe andina. La Paz.

STEINICKE, E. \& Vavti, S. (2006): Biographie, Identität und ethnische Vielfalt: Bedrohung und Chancen im Kanaltal (Italien). Europa Ethnica, 63: 12-20.

STETSON, G. (2012): Oil Politics and Indigenous Resistance in the Peruvian Amazon: Th e Rhetoric of Modernity against the Reality of Coloniality. Journal of Environment and Development 21 (1): 76-97 
THIMM, A. (2001): Ethnisch-politische Probleme der Modernisierung in den peruanischen Anden. - In: Th imm, A. (Hrsg.): Ethnische Konfl ikte in der Dritten Welt, Ursachen und Konsequenzen, Mainz, 189-208.

TRIVELLI, C. \& ESCOBAL, J. \& Revesz, B. (2009): Desarrollo rural en la sierra: aportes para el debate. Estudios de la Sociedad Rural, 37 - Diagnóstico y Propuesta, 44. Lima.

URTEAGA, P. \& Boelens, R. (Hrsg.) (2006): Derechos colectivos y políticas hídricas en la región andina. Lima.

WENCELIDES, B. (1986): Tiroler Bauern in Peru - Die Gründung der Kolonie Pozuzo und ihre Entwicklung bis heute. München, 95-98.

Martina Neuburger. Universität Hamburg Institut für Geographie. martina.neuburger@uni-hamburg.de

Katrin Singer. Universität Hamburg Institut für Geographie. katrin.singer@uni-hamburg.de 